\title{
The Nonlinear Absorption Coefficient for Direct Two-Photon Creation of Biexciton
}

\author{
W. UNGIER AND M. SUFFCZYŃSKI \\ Institute of Physics, Polish Academy of Sciences \\ al. Lotników 32/46, 02-668 Warszawa, Poland
}

(Received November 9, 2001; in final form December 18, 2001)

\begin{abstract}
The absorption coefficient for the two-photon creation of biexciton is calculated for $\mathrm{CuCl}$ and wurtzite crystals. Because of the resonance effect only the intermediate lowest optically active excitonic states are taken into account. The absorption coefficient is expressed by a functional of the biexciton envelope. The numerical results are computed with the envelope function of Hylleraas-Ore type (modified and minimized by Brinkman et al). The obtained results for $\mathrm{CuCl}$ are in good agreement with absorption measurements published by Gale and Mysyrowicz.
\end{abstract}

PACS numbers: 71.35.-y

\section{Probability of two-photon absorption}

The excitonic molecule, called the biexciton, is an elementary excitation in semiconductors or insulators, consisting of two electrons and two holes. The existence of this complex was confirmed by the emission and by the absorption spectra [1-4]. In the absorption spectrum a direct creation of the biexciton can be observed. The excitation of the system occurs from the ground state to the molecular state via a two-photon absorption process in an intense monochromatic radiation [3]. The emission lines are attributed to the radiative decomposition of biexcitons formed from the pool of high density free carriers generated in the system. The transition probability for the two-photon excitation of an insulating crystal from its ground state $|g\rangle$ to the state $|\mathrm{biex}\rangle$ with an excitonic molecule [5] is given by 


$$
W(\hbar \omega)=\frac{2 \pi}{\hbar} A^{2}\left|\left\langle\operatorname{biex}\left|\varepsilon \sum_{j} \boldsymbol{P}_{j} \frac{|i\rangle\langle i|}{E_{i g}-\hbar \omega} \varepsilon \sum_{j^{\prime}} \boldsymbol{P}_{j^{\prime}}\right| g\right\rangle\right|^{2} \delta\left(2 \hbar \omega-E_{\mathrm{biex}}\right),
$$

where $A=(e / m)^{2}(2 \pi \hbar N / \kappa \omega V), N$ is the number of photons in the incident beam with polarization $\varepsilon$ and frequency $\omega$ and $\boldsymbol{K}$ - the wave number vector of light, $\kappa$ is a dielectric constant for frequency $\omega, V$ is the volume of the crystal and $\boldsymbol{P}_{j}=\exp \left(\mathrm{i} \boldsymbol{K} \boldsymbol{r}_{j}\right) \boldsymbol{p}_{j}$ with $\boldsymbol{p}_{j}$ - the momentum and $\boldsymbol{r}_{j}$ - the position of the $j$-th electron.

According to Hanamura [6] only the resonant exciton intermediate states $|i\rangle$ are considered. In both cases, in $\mathrm{CuCl}$ and in wurtzite crystals, the optically active lowest exciton intermediate states have symmetry $\Gamma_{5}$ (in the notation of Koster et al. [7]). In $\mathrm{CuCl}$, the crystal of tetrahedral symmetry $T_{d}$, there exist three $\Gamma_{5}$ exciton states $J_{z}= \pm 1,0$ corresponding to a total angular momentum state $J=1$. In the wurtzite structure of uniaxial symmetry $C_{6 v}$ there exist only two $\Gamma_{5}$ exciton states with $J_{z}= \pm 1$. Because of the uniaxial symmetry of the wurtzite crystal these states are no more eigenstates of total $J$ but only of $J_{z}$.

In the $\mathrm{CuCl}$ crystal at the bottom $k=0$ of the conduction band the Bloch functions are of the form

$$
c_{1 / 2}=\phi_{c}|\uparrow\rangle \text { and } c_{-1 / 2}=\phi_{c}|\downarrow\rangle,
$$

where $\phi_{\mathrm{c}}$ can be identified with $4 s$-function of $\mathrm{Cu}$. At the top $\boldsymbol{k}=0$ of the valence band the Bloch functions are

$$
v_{1 / 2}=\psi_{1}|\downarrow\rangle+\psi_{0}|\uparrow\rangle \quad \text { and } \quad v_{-1 / 2}=\psi_{-1}|\uparrow\rangle-\psi_{0}|\downarrow\rangle
$$

with

$$
\psi_{ \pm 1}=(1 / \sqrt{3})\left(p_{x} \pm \mathrm{i} p_{y}\right) \quad \text { and } \quad \psi_{0}=(1 / \sqrt{3}) p_{z} .
$$

The top valence functions may be identified with $3 p$-functions of $\mathrm{Cl}$ (the atomic orbitals $p_{x}, p_{y}$, and $p_{z}$ are separately normalized).

In the wurtzite structure at the bottom $k=0$ the conduction Bloch functions are of the form defined in Eq. (2) and at the top $k=0$ of the valence band the Bloch functions are

$$
v_{3 / 2}=\psi_{1}|\uparrow\rangle \quad \text { and } \quad v_{-3 / 2}=\psi_{-1}|\downarrow\rangle
$$

with

$$
\psi_{ \pm 1}=(1 / \sqrt{2})\left(p_{x} \pm i p_{y}\right)
$$

For example, in CdS the conduction Bloch function $\phi_{c}$ is identified with $5 s$-function of $\mathrm{Cd}$ and $p_{x}, p_{y}$ are, to a good approximation, two of the $2 p$-functions of $\mathrm{S}$.

The matrix elements between the ground state $|g\rangle$ of the system and the $\Gamma_{5}$ exciton states [8] are expressed by the exciton envelope $\phi_{\mathrm{ex}}(\boldsymbol{r})$, a function of the electron-hole distance $r=\left|r_{1}-r_{2}\right|$

$$
\left\langle\operatorname{ex} \Gamma_{5}|\varepsilon p| g\right\rangle=\sqrt{V} \phi_{\mathrm{ex}}(0) \begin{cases}\left\langle\phi_{\mathrm{c}}|\varepsilon p| \psi_{ \pm 1}\right\rangle & \text { for } J_{z}= \pm 1 \\ \sqrt{2}\left\langle\phi_{\mathrm{c}}|\varepsilon p| \psi_{ \pm 1}\right\rangle & \text { for } J_{z}=0\end{cases}
$$


We use the long-wave approximation and thus we neglect the translational momentum $\boldsymbol{K}$ of exciton and momentum $2 \boldsymbol{K}$ of biexciton, where $\boldsymbol{K}$ is the wave number vector of light.

The matrix elements between $\Gamma_{5}$ exciton states and the biexciton ground state of the symmetry $\Gamma_{1}$ [8] are evaluated with the biexciton envelope $\psi_{\text {mol }}$ as follows:

$$
\left\langle\operatorname{biex} \Gamma_{1}|\varepsilon p| \operatorname{ex} \Gamma_{5}\right\rangle=I\left[\phi_{\mathrm{ex}}, \psi_{\mathrm{mol}}\right] \begin{cases}\left\langle\phi_{\mathrm{c}}|\varepsilon p| \psi_{ \pm 1}\right\rangle & \text { for } \quad J_{z}= \pm 1 \\ \sqrt{2}\left\langle\phi_{\mathrm{c}}|\varepsilon p| \psi_{0}\right\rangle & \text { for } \quad J_{z}=0,\end{cases}
$$

where the functional $I$ is the integral [9]

$$
I\left[\phi_{\mathrm{ex}}, \psi_{\mathrm{mol}}\right]=\iint \mathrm{d}^{3} x \mathrm{~d}^{3} y \psi_{\mathrm{mol}}^{*}(\boldsymbol{x}-\boldsymbol{y}, \boldsymbol{x}, \boldsymbol{y} / 2) \phi_{\mathrm{ex}}(\boldsymbol{y})
$$

with $\psi_{\text {mol }}\left(\boldsymbol{r}, \boldsymbol{r}^{\prime}, \boldsymbol{R}\right)$ a function $[10,11]$ of $\boldsymbol{r}=\boldsymbol{r}_{3}-\boldsymbol{r}_{1}, \boldsymbol{r}^{\prime}=\boldsymbol{r}_{4}-\boldsymbol{r}_{2}$ and $\boldsymbol{R}=$ $\left(r_{1}+r_{3}-r_{2}-r_{4}\right) / 2$. Here 1, 3 refer to the electrons, 2, 4 refer to the holes. Inserting matrix elements (7-8) into Eq. (1) we get

$$
\begin{aligned}
& W(\hbar \omega)=\frac{2 \pi}{\hbar} A^{2} \Phi_{\mathrm{ex}}^{2}(0) V I^{2}\left[\phi_{\mathrm{ex}}, \psi_{\mathrm{mol}}\right] \frac{\left|\left\langle\phi_{\mathrm{c}}\left|p_{x}\right| p_{x}\right\rangle\right|^{4}}{\left[E_{\mathrm{ex}}\left(\Gamma_{5}\right)-\hbar \omega\right]^{2}} \\
& \quad \times \delta\left(2 \hbar \omega-E_{\mathrm{biex}}\left(\Gamma_{1}\right)\right) \begin{cases}(4 / 9) \varepsilon^{2} & \text { for } \mathrm{CuCl}, \\
\left(\varepsilon_{x}^{2}+\varepsilon_{y}^{2}\right) & \text { for wurtzite crystals. }\end{cases}
\end{aligned}
$$

The energies $E_{\mathrm{ex}}$ and $E_{\mathrm{biex}}$ are measured from the level of the ground state $|g\rangle$. To compare the theoretical result of Eq. (10) with the experimentally observed total probability of the absorption process, we have to average $W(\hbar \omega)$ over the initial states. If the source of radiation has a spectral width $\Delta \omega$, we have to take the integral

$$
W_{\text {tot }}\left(\hbar \omega_{0}\right)=(\Delta \omega)^{-1} \int_{\omega_{0}-\Delta \omega / 2}^{\omega_{0}+\Delta \omega / 2} W(\hbar \omega) \mathrm{d} \omega
$$

where we assumed $\hbar \omega_{0}=E_{\text {biex }}\left(\Gamma_{1}\right) / 2$.

The absorption coefficient $\alpha\left(\hbar \omega_{0}\right)$ [5] is defined as the probability of the absorption of $\omega_{0}$-photon per unit length of its passage through the crystal and is related to $W_{\text {tot }}\left(\hbar \omega_{0}\right)$

$$
\alpha\left(\hbar \omega_{0}\right)=2 W_{\text {tot }}\left(\hbar \omega_{0}\right) /(N c / \sqrt{\kappa}),
$$

where $c / \sqrt{\kappa}$ is the velocity of light inside the crystal. The factor 2 in Eq. (12) is due to the number of $\omega_{0}$-photons absorbed in one process. Thus, if the source of the intense radiation is a laser of tunable frequency, for $\hbar \omega_{0}$ corresponding to one-half of biexciton energy we can expect to observe the two-photon absorption peak due to the creation of excitonic molecules. 


\section{Results and discussion}

The measurement of the absorption coefficient due to the direct biexciton creation is in the majority of crystals practically impossible: the absorption line corresponding to the two-photon creation of biexciton and the line corresponding to the creation of two free excitons differ in position by an amount comparable to the line width. The difference between the maxima of these lines is equal

$$
\Delta(\hbar \omega)=E_{\mathrm{ex}}-E_{\mathrm{biex}} / 2=E_{\mathrm{biex}}^{(\mathrm{b})} / 2+\Delta_{\mathrm{ex}}^{(\mathrm{exch})},
$$

where $E_{\mathrm{biex}}^{(\mathrm{b})}$ is the binding energy of the biexciton and $\Delta_{\mathrm{ex}}^{(\mathrm{exch})}$ is the energy of exchange splitting in exciton. In the $\mathrm{CuCl}$ crystal $\Delta_{\mathrm{ex}}^{(\mathrm{exch})}=E_{\mathrm{ex}}\left(\Gamma_{5}\right)-E_{\mathrm{ex}}\left(\Gamma_{2}\right)$ and in the wurtzite crystals $\Delta_{\text {ex }}^{(\text {exch })}=E_{\text {ex }}\left(\Gamma_{5}\right)-E_{\text {ex }}\left(\Gamma_{6}\right)$. The exciton exchange splitting in $\mathrm{CuCl}$ is $\Delta_{\mathrm{ex}}^{(\text {exch })}=6.2 \mathrm{meV}$ and in $\mathrm{CdS} \Delta_{\mathrm{ex}}^{(\text {exch })}=1.3 \mathrm{meV}$. In $\mathrm{CdS}$ $E_{\text {biex }}^{(\mathrm{b})} \cong 4.4 \mathrm{meV}$ and in CdSe $E_{\text {biex }}^{(\mathrm{b})}=1.2 \mathrm{meV}$. In $\mathrm{CuCl}$ due to the large mass of the hole, the biexciton binding energy $E_{\mathrm{biex}}^{(\mathrm{b})} \cong 30 \mathrm{meV}$ is much greater than in the wurtzite CdS and CdSe crystals.

Thus the large values of the binding energy and of the exchange splitting make possible observation of the biexcitons in $\mathrm{CuCl}$ to be distinguished from the state consisting of two noninteracting free excitons.

Using the intense monochromatic blue radiation from a tunable dye laser, of intensity $200 \mathrm{~kW}$, spectral width $6 \mathrm{~cm}^{-1}$ and pulse duration $16 \times 10^{-9} \mathrm{sec}$, Gale and Mysyrowicz [3] measured directly the two-photon absorption cross section in $\mathrm{CuCl}$ at several energies in the region around $E=2 \hbar \omega=2 E_{\mathrm{ex}}-E_{\text {biex }}^{(\mathrm{b})}$. They estimated the peak value of two-photon cross section to be $\delta \cong 10^{-42} \mathrm{~cm}^{4} \mathrm{~s}$ leading to a nonlinear absorption coefficient of the order $10^{5} \mathrm{~cm}^{-1}$ with light flux of $20^{25}$ photons $/ \mathrm{cm}^{2}$. Gale and Mysyrowicz interpreted their results for the absorption coefficient as being in good agreement with the estimation given by Hanamura [6].

Here we present results of our calculations of the two-photon absorption coefficient based on the optimized biexciton envelope function and the matrix element of the biexciton-exciton transition expressed by the biexciton and the free exciton envelopes [9]. The biexciton envelope used in our calculations was a Hylleraas-Ore [12] wave function modified by Brinkman et al. [10] and the free exciton envelope was the hydrogen-like $1 s$ wave function.

The values of the integral $I\left[\phi_{\mathrm{ex}}, \psi_{\mathrm{mol}}\right]$ were calculated for several mass ratios $\sigma=m_{\mathrm{e}} / m_{\mathrm{h}}$ [9]. Inserting into the formulae (10-12) the following data for $\mathrm{CuCl}$ : $\sigma=0.02, I \cong 4,\left|\left\langle\phi_{\mathrm{c}}\left|p_{x}\right| p_{x}\right\rangle\right|^{2} / m \cong 3 \mathrm{eV}[6], \hbar \omega \cong 3.2 \mathrm{eV}$ with the parameters of laser radiation used in the actual experiment [3], corresponding to the photon density $N / V=0.33 \times 10^{15}$ photons $/ \mathrm{cm}^{3}$, we get for the two-photon absorption coefficient $\alpha\left(\hbar \omega_{0}\right)=0.9 \times 10^{4} \mathrm{~cm}^{-1}$. The obtained result compared to previous estimation $\alpha\left(\hbar \omega_{0}\right)=2 \times 10^{6} \mathrm{~cm}^{-1}$, which corresponds to the probability of two-photon absorption calculated by Hanamura $[6,13]$, is nearer to the actual 
experimental value $\alpha=10^{5} \mathrm{~cm}^{-1}$. The biexciton-exciton dipole transition matrix element estimated in [6] corresponds to the biexciton radiative lifetime $\tau \cong 0.5 \mathrm{ps}$ in CdS, while the observed lifetime amounts to a few picoseconds [9, 14], thus the value of the biexciton-exciton matrix element given by Hanamura is definitely too large. Hanamura estimated it with a simple model of biexciton envelope which was not optimized, and without the formula for the integral $I\left[\phi_{\mathrm{ex}}, \psi_{\mathrm{mol}}\right]$ useful for a general case of the biexciton and exciton envelopes. The calculated value of the integral $I$ for CuCl [9] is about ten times smaller than the corresponding result obtained by Hanamura.

In wurtzite CdS the two-photon direct creation of biexcitons has been confirmed experimentally [4], but the absorption coefficient due to this transition has not been measured. The two-photon absorption line and the line due to the creation of two free excitons are closer in frequency than their linewidth. We calculated the two-photon absorption coefficient for CdS taking $\sigma=0.21, I \cong 10$, $\left|\left\langle\phi_{\mathrm{c}}\left|p_{x}\right| p_{x}\right\rangle\right|^{2} / m \cong 3 \mathrm{eV}[6], \hbar \omega_{0} \cong 2.5 \mathrm{eV}$ and $N / V=0.33 \times 10^{15}$ photons $/ \mathrm{cm}^{3}$. For the light polarization $\varepsilon \perp c$-axis we obtained $\alpha\left(\hbar \omega_{0}\right)=5 \times 10^{5} \mathrm{~cm}^{-1}$, which is our estimation of the contribution of the two-photon biexciton creation process to the optical absorption spectrum of CdS. The large value of the two-photon absorption coefficient for $\mathrm{CdS}$ compared with $\mathrm{CuCl}$ results mainly from a large value of the integral $I$ and from a small value of the resonance denominator $E_{\mathrm{ex}}\left(\Gamma_{5}\right)-\hbar \omega=3.5 \mathrm{meV}$ for CdS, while $E_{\mathrm{ex}}\left(\Gamma_{5}\right)-\hbar \omega=22 \mathrm{meV}$ for $\mathrm{CuCl}$.

\section{Acknowledgments}

The authors acknowledge a useful conversations with Prof. J. Mostowski.

\section{References}

[1] S. Shionoya, H. Saito, E. Hanamura, O. Akimoto, Solid State Commun. 12, 223 (1973).

[2] A. Mysyrowicz, J.B. Grun, R. Levy, A. Bivas, S. Nikitine, Phys. Lett. A 26, 615 (1968).

[3] G.M. Gale, A. Mysyrowicz, Phys. Rev. Lett. 32, 727 (1974).

[4] Y. Nozue, T. Itoh, M. Ueta, J. Phys. Soc. Jpn. 44, 1305 (1978).

[5] M. Inoue, Y. Toyozawa, J. Phys. Soc. Jpn. 20, 363 (1965).

[6] E. Hanamura, Solid State Commun. 12, 951 (1973).

[7] K.F. Koster, J.O. Dimmock, R.G. Wheeler, H. Statz, Properties of the Thirty Two-Point Groups, M.I.T. Press, Cambridge (Massachusetts) 1963.

[8] W. Ungier, P. Janiszewski, M. Suffczyński, Acta Phys. Pol. A 98, 411 (2000).

[9] W. Ungier, Solid State Commun. 110, 639 (1999).

[10] W.F. Brinkman, T.M. Rice, B. Bell, Phys. Rev. B 8, 1570 (1973).

[11] W. Ungier, M. Suffczyński, Int. J. Quant. Chem. 60, 1001 (1996). 
[12] E.A. Hylleraas, A. Ore, Phys. Rev. 71, 493 (1947).

[13] M. Ueta, H. Kanzaki, K. Kobayashi, Y. Toyozawa, E. Hanamura, in: Excitonic Processes in Solids, Springer, Berlin 1986, p. 63.

[14] D. Birdekal, J. Singh, V.G. Lyssenko, J. Erland, J.M. Hvam, Phys. Rev. Lett. 76, 672 (1996). 